\title{
The association between the anatomical features of renal tumours and the functional outcomes of robot-assisted partial nephrectomy
}

\author{
Jeong Woo Lee, MD; ${ }^{*}$ Sung Yong Cho, MD; Chanhoo Jeon, MD; Kyungtae Ko, MD,; \\ Hyeon Hoe Kim, MD, PhD
}

*Department of Urology, Sanggye Paik Hospital, Inje University College of Medicine, Dongil-ro, Nowon-gu, Seoul, Republic of Korea; †Department of Urology, SMG-SNU Boramae Medical Center, Dongjak-gu, Seoul, Republic of Korea; §Department of Urology, Seoul National University College of Medicine, Daehak-ro, Jongno-gu, Seoul, Republic of Korea

Cite as: Can Urol Assoc J 2014;8(11-12):e810-4. http://dx.doi.org/10.5489/cuaj.1642 Published online November 24, 2014.

\section{Abstract}

Introduction: We evaluated the the association between PADUA scores and postoperative renal function (after robot-assisted partial nephrectomy [RAPN]) and between PADUA scores and warm ischemic time (during RAPN).

Methods: We reviewed the clinical records of 106 patients who underwent RAPN for a single localized renal tumour between April 2009 and June 2012. Postoperative renal function was evaluated using estimated glomerular filtration rate (eGFR) in 85 patients who were followed for at least 6 months. PADUA scores for renal tumours were calculated using contrast-enhanced computed tomography images, if needed, along with magnetic resonance images in some cases.

Results: A PADUA score $\geq 10$ and WIT $\geq 30$ minutes were observed in $18(17.0 \%)$ and 51 (48.1\%) cases, respectively. PADUA scores were significantly correlated with WIT $(p=0.019)$ and percent change in eGFR at 6 months postoperatively $(p=0.005)$. PADUA score (continuous variable, odds ratio [OR] 1.694, $p=0.007$ ) and the high-risk group (PADUA score $\geq 10$ ) (OR 5.429; $p=0.020$ ) were significantly associated with a WIT of $\geq 30$ minutes by multivariate analysis. A 1-point increase in the PADUA score was associated with an eGFR decrease of $>20 \%$ at 6 months after RAPN (OR 1.799; $p=0.076)$. In addition, a PADUA score $\geq 10$, or high risk, (OR 13.965; $p=0.003$ ) was an independent predictor of an eGFR decrease of $>20 \%$ at 6 months after RAPN.

Conclusions: The PADUA classification can reliably predict WIT and postoperative renal functional outcome after RAPN. Furthermore, the study suggests that anatomical aspects of renal tumours are associated with functional outcome after RAPN.

\section{Introduction}

Clinical tumour size and tumour anatomical features are the main considerations in planning surgery for localized renal tumours. The preoperative aspects and dimensions used for an anatomical (PADUA) classification provide a standardized anatomical system for surgical decision-making. PADUA scores also enable us to predict the risk of overall complications in patients scheduled for open partial nephrectomy. ${ }^{1}$ PADUA scores have been validated to predict warm ischemic time (WIT) and perioperative complications for open, laparoscopic, and robot-assisted partial nephrectomy (RAPN). ${ }^{2-6}$

Functional outcome is an important parameter during the surgical planning of renal tumours. Numerous welldocumented variables have been associated with postoperative renal function. ${ }^{7}$ In particular, tumour size and WIT are important predictors of renal function deterioration after partial nephrectomy. ${ }^{7-9}$ However, there is little data available regarding the relationship between functional outcomes and the anatomical features of renal tumours treated by RAPN.

In this study, we examined the associations between PADUA scores and WITs and postoperative renal function in consecutive patients treated by RAPN for a single, unilateral renal tumour.

\section{Methods}

We retrospectively evaluated 106 consecutive patients who underwent RAPN for a localized renal tumour performed by a single surgeon, at our institution between April 2009 and June 2012. All cases were performed in the same way using a transperitoneal approach. Postoperative renal function was evaluated in 85 of these 106 patients who were followed up for at least 6 months. Patients with a solitary kidney or who underwent radical conversion were excluded from the 
Anatomical features of renal tumour and functional outcomes

analysis of functional outcomes. The study was approved by the institutional review board of Seoul National University Hospital.

The following variables were collected for all patients: age, gender, body mass index (BMI), American Society of Anesthesiologists (ASA) score, laterality, and clinical tumour size. All patients underwent preoperative laboratory tests, such as serum hemoglobin, creatinine $(\mathrm{SCr})$, coagulation profile, and urinalysis. SCr was measured before and after RAPN in all patients. The estimated glomerular filtration rate (eGFR) was calculated using the 4-variable IDMSMDRD (Isotope Dilution Mass Spectrometry-Modification of Diet in Renal Disease) formula (eGFR $=175 \times \mathrm{SCr}^{-1.154}$ $\times$ age $^{-0.203} \times 0.742$ [if female]) preoperatively and at 6 months postoperatively.

Renal tumours were preoperatively evaluated by contrastenhanced computed tomography (CT) using a 3-mm axial slice thickness, and when needed by magnetic resonance imaging (MRI). Based on retrospective examinations of all radiologic images, 2 urologists assigned anatomical parameters to each tumour according to the PADUA classification. ${ }^{1}$ Scores for each of parameter were then totaled. Both urologists were unaware of the final outcomes. Patients were stratified into 3 groups based on PADUA scores: to low-risk (6-7), intermediate-risk (8-9), and high-risk ( $\geq 10)$.

Patients were compared with regard to perioperative parameters, including console time, WIT, estimated blood loss (EBL), length of hospital stay, and change in eGFR at 6 months postoperatively. The primary end point in this study was percent change in eGFR ([eGFR followup $\left.-\mathrm{eGFR}_{\text {preoperative }}\right] /$ $\mathrm{eGFR}_{\text {preoperative }} \times 100 \%$ ).

\section{Statistical analysis}

Clinical parameters were presented as medians and interquartile ranges (IQRs). The Kruskal-Wallis test was used to examine the correlation between PADUA scores and perioperative outcomes. The association between PADUA scores and WIT $\geq 30$ minutes and an eGFR decrease of $>20 \%$ at 6 months postoperatively were analyzed using logistic regression models and resultswere presented as odds ratios (OR) and $95 \%$ confidence intervals (Cls). Statistical analyses were performed using SPSS ver. 19.0 (IBM, Armonk, NY). Statistical significance was set at $p<0.05$.

\section{Results}

In total, 106 tumours treated by RAPN were evaluated in this study (Table 1). Seventy-five (70.8\%) patients were male and $3(2.8 \%)$ patients had a solitary kidney. The median age at RAPN was 53 (IQR 45-59), and the median BMI was $24.9 \mathrm{~kg}$ / $\mathrm{m}^{2}$ (IQR 22.4-26.8). Forty-nine (46.2\%) tumours were located at the right kidney. Twenty-nine $(27.4 \%)$ patients had a

\begin{tabular}{lc} 
Table 1. Demographics of the study cohort (n = 106) \\
\hline Age, year (median, IQR) & $53(45-59)$ \\
Male patients, no. (\%) & $75(70.8)$ \\
BMI, kg/m² (median, IQR) & $24.9(22.4-26.8)$ \\
ASA score (median, IQR) & $1(1-2)$ \\
Right side, no. (\%) & $49(46.2)$ \\
Solitary kidney, no. (\%) & $3(2.8)$ \\
Previous abdominal surgery, no. (\%) & $29(27.4)$ \\
Preoperative Hb, g/dL (median, IQR) & $14.3(13.1-15.3)$ \\
Preoperative creatinine, mg/dL (median, IOR) & $0.9(0.7-1.0)$
\end{tabular}

Preoperative eGFR, $\mathrm{mL} / \mathrm{min}$ per $1.73 \mathrm{~m}^{2}$ (median, IQR)

$83.40(70.9-91.2)$

Clinical tumour size, $\mathrm{cm}$ (median, IQR)

PADUA score (median, IQR)

$2.1(1.5-2.9)$ $8(7-9)$

BMI: body mass index; ASA: American Society of Anesthesiology; Hb: hemoglobin; eGFR: estimated glomerular filtration rate; IQR: interquartile interval; PADUA: preoperative aspects and dimensions used for an anatomical.

history of abdominal or pelvic surgery. The median preoperative eGFR was $83.40 \mathrm{~mL} / \mathrm{min}$ per $1.73 \mathrm{~m}^{2}$ (IQR 70.9-91.2), and the median SCr was $0.9 \mathrm{mg} / \mathrm{dL}$ (IQR 0.7-1.0). Based on preoperative CT scans, the median tumour size was $2.1 \mathrm{~cm}$ (IQR 1.5-2.9) and the median PADUA score was 8 (IQR 7-9). According to the risk-group stratification proposed in a previous study, $32(30.2 \%), 56(52.8 \%)$, and $18(17.0 \%)$ were included in the low- (6-7), intermediate- (8-9), and high-risk (10-13) groups, respectively.

We tallied the perioperative outcomes (Table 2). The median console time was 135 minutes (IQR 110-158), median WIT 29.0 minutes (IQR 23-36) and median EBL $200 \mathrm{~mL}$ (IQR 100-300). The median length of hospital stay was 3 days (IQR $3-4)$. Six months after RAPN, a median decline of $-6.30 \mathrm{~mL} / \mathrm{min}$ per $1.73 \mathrm{~m}^{2}$ (IQR -13.4 to 2.6) was observed for eGFR and a median increase of $0.07 \mathrm{mg} / \mathrm{dL}$ (IQR -0.02 to 0.12) for SCr.

PADUA scores were significantly correlated with WITs $(p=0.019)$ and percent changes in eGFR at 6 months postoperatively $(p=0.005)$. However, no significant correlation was found between PADUA scores and console times $(p=0.306), \mathrm{EBL}(p=0.264)$, or length of hospital stay $(p=0.420)($ Table 3$)$.

\begin{tabular}{lc}
\hline Table 2. Perioperative outcomes & \\
\hline Intraoperative variables & Median (IQR) \\
\hline Console time, min & $135(110-158)$ \\
Warm ischemic time, min & $29.0(23-36)$ \\
Estimated blood loss, $\mathrm{mL}$ & $200(100-300)$ \\
\hline Postoperative variables & Median (IQR) \\
\hline Hospital stay, day & $3(3-4)$ \\
Postoperative change in $\mathrm{Hb}, \mathrm{g} / \mathrm{dL}$ & $-1.30(-2.1$ to -0.7$)$ \\
6-month change in creatinine, $\mathrm{mg} / \mathrm{dL}$ & $0.07(-0.02$ to 0.12$)$ \\
6-month change in eGFR, $\mathrm{mL} / \mathrm{min}$ per $1.73 \mathrm{~m}^{2}$ & $-6.30(-13.4$ to 2.6$)$ \\
\hline Hb: hemoglobin; eGFR: estimated glomerular filtration rate; IQR: interquartile interval. \\
\hline
\end{tabular}


Lee et al.

\begin{tabular}{lcccccc}
\hline \multicolumn{7}{l}{ Table 3. Correlation between PADUA score and perioperative outcomes } \\
\hline PADUA score & WIT & Console time & EBL & Change in eGFR & Hospital stay & Percent change in eGFR \\
\hline Low (6-7) & $28.1(7.9)$ & $130.1(38.7)$ & $284.1(50.2)$ & $-2.75(12.9)$ & $3.2(0.8)$ & $-4.32(13.1)$ \\
Intermediate (8-9) & $31.2(9.6)$ & $140.1(39.0)$ & $270.4(246.4)$ & $-4.10(10.4)$ & $3.4(1.2)$ & $-4.14(12.3)$ \\
High (10-13) & $37.7(6.9)$ & $138.9(18.2)$ & $372.8(265.1)$ & $-16.4(22.8)$ & $3.7(2.2)$ & $-17.56(23.0)$ \\
$p$ value & 0.019 & 0.306 & 0.264 & 0.005 & 0.420 & 0.005
\end{tabular}

PADUA score: preoperative aspects and dimensions used for an anatomical score (longitudinal location, exophytic rate, renal rim location, involvement of the renal sinus or the urinary collecting system, and tumor size); WIT: warm ischemic time; EBL: estimated blood loss; eGFR: estimated glomerular filtration rate.

PADUA score (treated as a continuous variable) was associated with a WIT of $\geq 30$ minutes (OR: 1.682 for each 1 -point increase; 95\% Cl, 1.181-2.394; $p=0.004)$. After adjusting for age, gender, BMI, laterality, and surgeon experience, multivariate regression analysis showed that PADUA score was significantly associated with a WIT of $\geq 30$ minutes (OR 1.694 for each 1-point increase; 95\% Cl, 1.155-2.485; $p=0.007)$. According to the risk-group stratification, high risk (PADUA score $\geq 10$ ) was an independent predictor of a WIT of $\geq 30$ minutes (OR 5.429; 95\% Cl, 1.309-22.517; $p=0.020$ ) by multivariate analysis (Table 4 ).
PADUA score (treated as a continuous variable) was significantly associated with an eGFR decrease of $>20 \%$ at 6 months after RAPN (OR 2.059 for each 1 point increase; $95 \% \mathrm{Cl}, 1.113-3.808 ; p=0.021)$. A 1 -point-increase in the PADUA score non-significantly increased the risk of an eGFR decrease of $>20 \%$ at 6 months after RAPN by multivariate analysis $(p=0.076)$. The high-risk group was significantly associated with an eGFR decrease of $>20 \%$ at 6 months after RAPN (OR 14.889; 95\% Cl, 3.158-70.203; $p=0.001$ ) by univariate analysis. After adjusting for age, gender, BMI, laterality, prevalence of hypertension or diabetes, surgeon

Table 4. Univariate and multivariate analyses for the prediction of WIT $\geq 30 \mathrm{~min}$ and an eGFR decrease of $>20 \%$

\begin{tabular}{|c|c|c|c|c|}
\hline \multirow{3}{*}{ Variable } & \multicolumn{4}{|c|}{ Warm ischemic time $\geq \mathbf{3 0} \mathbf{m i n}$} \\
\hline & \multicolumn{2}{|c|}{ Univariate } & \multicolumn{2}{|c|}{ Multivariate } \\
\hline & OR (95\% Cl) & $p$ value & OR (95\% Cl) & $p$ value \\
\hline Age (continuous) & $0.985(0.952-1.019)$ & 0.379 & $0.983(0.945-1.024)$ & 0.414 \\
\hline Gender (male ref.) & $0.703(0.298-1.659)$ & 0.421 & $1.102(0.412-2.950)$ & 0.847 \\
\hline BMI (continuous) & $0.824(0.715-0.951)$ & 0.008 & $0.857(0.728-1.010)$ & 0.065 \\
\hline Laterality (Rt. ref.) & $0.822(0.375-1.800)$ & 0.624 & $0.909(0.375-2.201)$ & 0.833 \\
\hline Experience (>30 ref.) & $2.182(0.087-5.366)$ & 0.089 & $1.854(0.676-5.080)$ & 0.230 \\
\hline \multicolumn{5}{|l|}{ PADUA risk stratification } \\
\hline \multicolumn{5}{|l|}{ Low (6-7) } \\
\hline Intermediate (8-9) & $2.154(0.852-5.448)$ & 0.105 & $2.350(0.890-6.202)$ & 0.085 \\
\hline High (10-13) & $6.500(1.679-25.162)$ & 0.007 & 5.429 (1.309-22.517) & 0.020 \\
\hline \multirow[t]{2}{*}{ PADUA (continuous)* } & $1.682(1.182-2.394)$ & 0.004 & $1.694(1.155-2.485)$ & 0.007 \\
\hline & \multicolumn{4}{|c|}{ eGFR decrease $>20 \%^{\dagger}$} \\
\hline \multirow[t]{2}{*}{ Variable } & \multicolumn{2}{|c|}{ Univariate } & \multicolumn{2}{|c|}{ Multivariate } \\
\hline & OR (95\% Cl) & $p$ value & OR (95\% Cl) & $p$ value \\
\hline Age (continuous) & $0.967(0.912-1.026)$ & 0.271 & $0.985(0.911-1.065)$ & 0.708 \\
\hline Gender (male ref.) & $1.020(0.235-4.419)$ & 0.979 & $0.823(0.123-5.504)$ & 0.840 \\
\hline BMI (continuous) & $0.793(0.620-1.014)$ & 0.064 & $0.822(0.593-1.138)$ & 0.238 \\
\hline Laterality (Rt. ref.) & $1.619(0.377-6.956)$ & 0.517 & $1.408(0.244-8.120)$ & 0.702 \\
\hline Hypertension (none ref.) & $2.160(0.420-11.113)$ & 0.357 & $0.745(0.063-8.871)$ & 0.816 \\
\hline Diabetes (none ref.) & $1.075(0.120-9.623)$ & 0.949 & $3.114(0.175-55.246)$ & 0.439 \\
\hline Experience (>30 ref.) & $1.773(0.427-7.036)$ & 0.442 & $1.379(0.235-8.092)$ & 0.722 \\
\hline WIT (continuous) & $1.022(0.947-1.103)$ & 0.568 & $0.977(0.872-1.094)$ & 0.682 \\
\hline \multicolumn{5}{|l|}{ PADUA risk stratification } \\
\hline \multicolumn{5}{|l|}{ Low \& Intermediate (6-9) } \\
\hline High (10-13) & 14.889 (3.158-70.203) & 0.001 & $13.965(2.430-80.262)$ & 0.003 \\
\hline PADUA (continuous) ${ }^{\ddagger}$ & $2.059(1.113-3.808)$ & 0.021 & $1.799(0.941-3.441)$ & 0.076 \\
\hline
\end{tabular}

WIT: warm ischemic time; eGFR: estimated glomerular filtration rate; OR: odds ratio; Cl: confidence interval; BMI: body mass index; ref.: reference; PADUA: preoperative aspects and

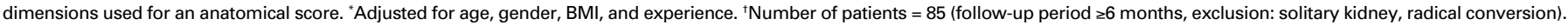

${ }^{\ddagger}$ Adjusted for age, gender, BMI, laterality, hypertension, diabetes, experience, and warm ischemic time. 
experience, and WIT, multivariate analysis showed that high risk (a PADUA score $\geq 10$ ) independently predicted an eGFR decrease of $>20 \%$ at 6 months after RAPN (OR 13.965; 95\% $\mathrm{Cl}, 2.430-80.262 ; p=0.003$ ) (Table 4).

\section{Discussion}

Nephron-sparing surgery (NSS), if technically feasible, is currently favoured compared to radical nephrectomy for localized renal tumours of $\leq 7 \mathrm{~cm}$ in terms of oncological and functional outcomes. ${ }^{10,11}$ However, NSS has been underused due to its technical difficulty. In addition to tumour size, other anatomical features, such as location and growth pattern, which also represent surgical complexity, play critical roles when assessing the feasibility of NSS to treat renal tumours. Three nephrometry scoring systems, namely the R.E.N.A.L., PADUA, and C-index, which are all based on the anatomical features of renal tumours, are standard tools for planning the surgical treatment of renal tumors and for facilitating patient comparisons. ${ }^{1,12,13}$ The PADUA classification was originally designed based on the radiological review findings of urologists, and consists of parameters easily recognized on $\mathrm{CT}$ or MRI. Moreover, with respect to the anatomical features of renal tumours, the PADUA system is more detailed than the other scoring systems. ${ }^{1}$ For these reasons, we decided to use the PADUA classification to evaluate the association between anatomical features and functional outcomes after RAPN.

Since it was first proposed as a means of predicting overall complications after open partial nephrectomy in 2009, the PADUA classification has been validated with respect to perioperative outcomes, such as complications and ischemic time..$^{2-6}$ However, only a few reports have evaluated the association between PADUA scores and functional outcomes. In a cohort of 101 patients who underwent laparoscopic partial nephrectomy, moderate- and high-risk groups (PADUA scores of $\geq 8$ ) exhibited greater percent changes in $\mathrm{SCr}$ between baseline and postoperative day 1, than the low-risk group. ${ }^{14}$ Bylund and colleagues concluded that PADUA scores are significantly associated with percent change in eGFR, despite a weak correlation. ${ }^{6}$ However, in the Bylund study nadir eGFR between 1 to 6 months after partial nephrectomy was used to evaluate functional outcomes, and this did not represent ultimate renal function. ${ }^{6}$ In addition, a number of surgical modalities via open, laparoscopic, and robot-assisted approaches were included which makes comparisons of renal functional outcomes difficult. Therefore, in the present study, we used preoperative and 6-month postoperative eGFR to assess the influence of PADUA score on ultimate renal function. We found that the PADUA score, particularly for the high-risk group, was independently associated with an eGFR decrease of $>20 \%$ at 6 months after RAPN (OR 13.965; $p=0.003)$. This result showed a strong correlation between the anatomical features of renal tumours and a deteriorating ultimate renal function in patients treated by RAPN for a localized renal tumour.

Ischemic time is considered an important surrogate of surgical complexity during partial nephrectomy. ${ }^{1,6}$ PADUA classification, which was not evaluated in the original study, has been externally validated for the association with prolonged ischemic time. PADUA score was significantly associated with WIT ( $p=0.016)$ for laparoscopic PN, but no correlation was found between WIT and risk groups categorized according to tumour complexity $(14.0,20.0$, and 22.5 minutes (median) for low-, intermediate-, and high-risk groups, respectively). ${ }^{14}$ In contrast to these results, in another study, in which 62 patients with PADUA scores of $<8$ and $\geq 8$ were compared, PADUA score independently predicted a WIT of $>20$ minutes after RAPN (OR 5.408; $p=0.002){ }^{3}$ In a multi-institutional study, Ficarra and colleagues found that patients in the intermediate- (OR 2.951; $p<0.001)$ and high-risk (OR 3.608; $p<0.001)$ groups were more likely to have a WIT of $>20$ minutes compared to patients in the low-risk group after RAPN. ${ }^{5}$ In the present study, PADUA score exhibited an OR of 1.694 for each 1-point increase $(p=0.007)$ and the high-risk group (OR: $5.429 ; p=0.020)$ independently predicted a WIT of $\geq 30$ minutes after adjusting for age, gender, BMI, laterality, and surgeon experience.

Other parameters of surgical complexity during NSS include operation time, blood loss, length of hospital stay, and overall complication rate. Previous studies have reported that PADUA scores are correlated with console times, $\mathrm{EBL}$, and overall complication rates in patients treated by RAPN. ${ }^{3,5}$ In contrast to these results, no significant relationship was found between PADUA scores and operation times, $\mathrm{EBL}$, hospital stays, or the occurrence of complications in a series of patients undergoing laparoscopic PN. ${ }^{14}$ In the present study, tumour complexity based on PADUA risk-group stratification was not correlated with console time, EBL, or hospital stay (Table 3), and overall complications were not associated with PADUA score or risk-group stratification (data not shown).

The present study has some shortcomings related to the non-randomized, retrospective study design, and its relatively small cohort of patients who underwent RAPN by a single surgeon at a single centre. We measured renal function by eGFR using the 4-variable IDMS-MDRD formula, instead of renal scintigraphy or measured GFR. The IDMS-MDRD formula was originally developed for the chronic kidney disease population. Retrospectively in our series, it was not possible to measure a change of renal function of a single operative kidney by renogram or removed volume data. The MDRD formula in partial nephrectomy has been used to provide an appropriate estimate of renal function despite its limitation application in the partial nephrectomy population (typical patients with a normal contralateral kidney). ${ }^{15}$ 
Lee et al.

The median WIT in the present study was 29.0 minutes. Recent reports demonstrate that the warm ischemia should be done by 20 minutes. ${ }^{7,16,17}$ However, it has been suggested that the safe margin of WIT is 30 minutes. ${ }^{18,19}$ Moreover, in retrospective studies, the warm ischemia of 40 to 55 minutes was safe and sufficient for the complete recovery of renal function. ${ }^{20,21}$ Lee and colleagues demonstrated that ultimate renal function decreases proportionally with increasing ischemia time in the warm and cold ischemia groups. ${ }^{22}$ Accordingly, we believe that our results provide information on the correlation between the anatomical features of renal tumours and the functional outcomes of RAPN. Furthermore, BMI IQR (22.4-26.8) was narrow in this study. Caucasians are more highly obese than Koreans. For example, the proportion of Korean adults (over 30) with a BMI $>25$ was $33.1 \%$ in $2008 .{ }^{23}$ Finally, oncological outcomes were not evaluated due to the short-term follow-up used. Accordingly, we suggest that prospective, randomized controlled trials be conducted to validate the PADUA classification in long-term oncological and functional outcomes in patients undergoing RAPN.

\section{Conclusion}

The PADUA classification reliably predicts WIT and postoperative renal functional outcomes after RAPN. A PADUA score of $\geq 10$ was associated with a 5.4-fold greater risk of a WIT of $\geq 30$ minutes and a 14 -fold greater risk of an eGFR decrease of $>20 \%$ at 6 months after RAPN. Renal functions after RAPN are significantly influenced by anatomical aspects of renal tumours.

Competing interests: Dr. Lee, Dr. Cho, Dr. Jeon, Dr. Ko and Dr. Kim all declare no competing financial or personal interests.

This paper has been peer-reviewed.

\section{References}

1. Ficarra V, Novara G, Secco S, et al. Preoperative aspects and dimensions Used for an anatomical (PADUA) classification of renal tumours in patients who are candidates for nephron-sparing surgery. Eur Urol 2009;56:786-93. http://dx.doi.org/10.1016/i.eururo.2009.07.040

2. Waldert $M$, Waalkes $S$, Klatte $T$, et al. External validation of the preoperative anatomical classification for prediction of complications related to nephron-sparing surgery. World J Urol 2010;28:531-5. http:// dx.doi.org/10.1007/s00345-010-0577-8

3. Mottrie A, Schatteman P, De Wil P, et al. Validation of the preoperative aspects and dimensions used for an anatomical (PADUA) score in a robot-assisted partial nephrectomy series. World J Urol 2013;31:799804. http://dx.doi.org/10.1007/s00345-010-0639-y. Epub 2011 Feb 1.
4. Tyritzis SI, Papadoukakis S, Katafigiotis L, et al. Implementation and external validation of Preoperative Aspects and Dimensions Used for an Anatomical (PADUA) score for predicting complications in 74 consecutive partial nephrectomies. BJU Int 2012;109:1813-8. http://dx.doi.org/10.1111/i.1464410X.2011.10644.X

5. Ficarra V, Bhayani S, Porter J, et al. Predictors of warm ischemia and perioperative complications in a multicenter, international series of robot-assisted partial nephrectomy. Eur Urol 2012;61:395-402. http://dx.doi.org/10.1016/i.eururo.2011.10.046

6. Bylund JR, Gayheart D, Fleming T, et al. Association of tumor size, location, R.E.N.A.L., PADUA and centrality index score with perioperative outcomes and postoperative renal function. J Urol 2012;188:1684-9. http://dx.doi.org/10.1016/i.juro.2012.07.043

7. Lane $B R$, Babineau $D C$, Poggio ED, et al. Factors predicting renal functional outcome after partial nephrectomy. J Urol 2008;180:2363-8. http://dx.doi.org/10.1016/i.juro.2008.08.036

8. Thompson RH, Lane BR, Lohse CM, et al. Renal function after partial nephrectomy: Effect of warm ischemia relative to quantity and quality of preserved kidney. Urology 2012;79:356-60. http://dx.doi. org/10.1016/i.urology.2011.10.031

9. Thompson RH, Lane BR, Lohse $\mathrm{CM}$, et al. Every minute counts when the renal hilum is clamped during partial nephrectomy. Eur Urol 2010;58:340-5. http://dx.doi.org/10.1016/i.eururo.2010.05.047

10. Crépel $M$, Jeldres $C$, Perrotte $P$, et al. Nephron-sparing surgery is equally effective to radical nephrectomy for TIBNOMO renal cell carcinoma: A population-based assessment. Urology 2010;75:271-5. http:// dx.doi.org/10.1016/i.urology.2009.04.098

11. Leibovich $B C$, Blute $M$, Cheville JC, et al. Nephron sparing surgery for appropriately selected renal cell carcinoma between 4 and $7 \mathrm{~cm}$ results in outcome similar to radical nephrectomy. J Urol 2004; 171:106670. http://dx.doi.org/10.1097/01.ju.0000113274.40885.db

12. Kutikov A, Uzzo RG. The R.E.N.A.L. nephrometry score: A comprehensive standardized system for quantitating renal tumor size, location and depth. J Urol 2009;182:844-53. http://dx.doi.org/10.1016/i. juro.2009.05.035

13. Simmons MN, Ching CB, Samplaski MK, et al. Kidney tumor location measurement using the C index method. J Urol 2010;183:1708-13. http://dx.doi.org/10.1016/i.juro.2010.01.005

14. Okhunov Z, Rais-Bahrami S, George AK, et al. The comparison of three renal tumor scoring systems: C-Index, P.A.D.U.A., and R.E.N.A.L. nephrometry scores. J Endourol 2011;25:1921-4. http://dx.doi. org/10.1089/end.2011.0301

15. Wszolek MF, Kenny PA, Lee Y, et al. Comparison of hilar clamping and non-hilar clamping partial nephrectomy for tumours involving a solitary kidney. BJU Int 2011;107:1886-92. http://dx.doi. org/10.1111/i.1464-410X.2010.09713.x

16. Thompson $\mathrm{RH}$, Leibovich $\mathrm{BC}$, Lohse $\mathrm{CM}$, et al. Complications of contemporary open nephron sparing surgery: A single institution experience. J Urol 2005;174:855-8. http://dx.doi.org/10.1097/01. ju.0000169453.29706.42

17. Thompson RH, Frank I, Lohse CM, et al. The impact of ischemia time during open nephron sparing surgery on solitary kidneys: A multi-institutional study. J Urol 2007;177:471-6. http://dx.doi.org/10.1016/i. juro.2006.09.036

18. Novick AC. Renal hypothermia: In vivo and ex vivo. Urol Clin North Am 1983;10:637-44.

19. Ward JP. Determination of the optimum temperature for regional renal hypothermia during temporary renal ischaemia. Br J Urol 1975;47:17-24. http://dx.doi.org/10.1111/i.1464-410X.1975.tb03913.x

20. Godoy G, Ramanathan V, Kanofsky JA, et al. Effect of warm ischemia time during laparoscopic partial nephrectomy on early postoperative glomerular filtration rate. J Urol 2009;181:2438-43, discussion 2443-5. http://dx.doi.org/10.1016/i.juro.2009.02.026

21. Bhayani SB, Rha KH, Pinto PA, et al. Laparoscopic partial nephrectomy: Effect of warm ischemia on serum creatinine. J Urol 2004;172:1264-6. http://dx.doi.org/10.1097/01.ju.0000138187.56050.20

22. Lee JW, Kim H, Choo M, et al. Different methods of hilar clamping during partial nephrectomy: Impact on renal function. Int J Urol 2014;21:232-6. http://dx.doi.org/10.1111/iju.12255

23. Ministry of Health, Welfare and Family Affairs. The Fourth Korea Nutritional Health and Nutrition Examination Surgery (KNHANES IV). Seoul, Korea. Ministry of Health, Welfare and Family Affairs; 2008.

Correspondence: Dr. Hyeon Hoe Kim, Department of Urology, Seoul National University College of Medicine, 101 Daehak-ro, Jongno-gu, Seoul, 110-744, Republic of Korea; hhkim@snu.ac.kr 